\title{
Corrigendum to: "Nonlocal effects in the optical response of composite materials with metallic nanoparticles" [Solid State Commun. 133 (2005) 315-320]
}

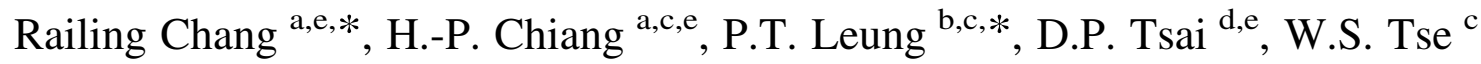 \\ a Institute of Optoelectronic Sciences, National Taiwan Ocean University, Keelung, Taiwan, ROC \\ ${ }^{\mathrm{b}}$ Department of Physics, Portland State University, P.O. Box 751, Portland, OR 97207-0751, USA \\ ${ }^{\mathrm{c}}$ Institute of Physics, Academia Sinica, Taipei, Taiwan, ROC \\ ${ }^{\mathrm{d}}$ Department of Physics, National Taiwan University, Taipei 10617, Taiwan, ROC \\ ${ }^{\mathrm{e}}$ Center of Nanostorage Research, National Taiwan University, Taipei 10617, Taiwan, ROC
}

Received 13 July 2004; received in revised form 30 September; accepted 6 November 2004 by S.G. Louie Available online 20 December 2005

After the publication of the above article, we have become aware of a key reference which had completely skipped our attention previously (even with the help of a 'key word' search using the science citation index). This is:

G.S. Agarwal and R. Inguva, Phys. Rev. B 30 (1984) 6108.

Agarwal and Inguva had essentially studied the same problem and obtained similar results as ours. The two models we have studied-Maxwell-Garnett and Bruggeman-are essentially contained in the two theories of theirs: the average-T-matrix approximation and coherent-potential approximation, respectively.
The only additional (relatively minor) things we have done in our paper include:

(i) Study of the nonlocal (NL) effects beyond the hydrodynamic model by using the Lindhard-Mermin RPA model; (ii) study of also the NL effects on dispersion (besides absorption) of the composite material; and (iii) elucidation of the mechanism for the appearance of resonance in absorption for large metallic volume fraction in the Bruggeman model caused by the NL effects.

We apologize for our unintentional omission of the work of Agarwal and Inguva.

DOI of original article: $10.1016 /$ j.ssc.2004.11.014

* Corresponding authors. Address: Institute of Optoelectronic Sciences, National Taiwan Ocean University, 2, Pei-Ning Road, Keelung, Taiwan, ROC. Tel.: + 886 24622192x6714; fax: +886 224634360.

E-mail address: rlchang@mail.ntou.edu.tw (R. Chang). 